\title{
Autosomal recessive limb-girdle muscular dystrophy type 20
}

INSERM

\section{Source}

INSERM. (1999). Orphanet: an online rare disease and orphan drug data base. Autosomal recessive limb-girdle muscular dystrophy type 20. ORPHA:206564

Autosomal recessive limb-girdle muscular dystrophy type 20 (LGMD2O) is a form of limb-girdle muscular dystrophy characterized by an onset in childhood or adolescence of rapidly progressive proximal limb muscle weakness (particularly affecting the neck, hip girdle, and shoulder abductors), hypertrophy in the calves and quadriceps, ankle contractures, and myopia. 